\title{
Rare variations of hepatic arteries in association with variable origin of gastroduodenal artery found in multidetector computed tomography angiography
}

\author{
A. Skórzewska1 ${ }^{1}$, P. Stajgis ${ }^{2}$, M. Grzymisławska ${ }^{1}$, M. Rojewska ${ }^{1}$, M. Krajecki ${ }^{1}$, \\ M. Bruska' ${ }^{1}$ R. Juszkat ${ }^{2}$ \\ ${ }^{1}$ Department of Anatomy, University of Medical Sciences, Poznan, Poland \\ ${ }^{2}$ Department of General and Interventional Radiology, University of Medical Sciences, Poznan, Poland \\ [Received 3 April 2014; Accepted 1 May 2014]
}

\begin{abstract}
Three rare anatomical variations were found during study on hepatic arterial vascularisation in multidetector computed tomography angiography. In the first described variation the common hepatic artery (CHA) arises from the celiac trunk (CTr) and supplies right hepatic lobe. The left lobe of the liver is supplied by aberrant left hepatic artery originating as a common trunk with the left gastric artery and the splenic artery. This variation may correspond to the type 2 in Michels' classification coexisting with one of three possible patterns of the CTr division (when the CHA is the first branch of the CTr and the gastrosplenic trunk is the second one). The second variation corresponds to the very early bifurcation of the CHA arising from the CTr. Both, the right and left hepatic arteries originate separately from the $C T r$. The gastroduodenal artery (GDA) originates from the left hepatic artery. It may be regarded as the variation of most common type 1 according to Michels. In the third case the CHA gives raise to the GDA and terminates as the right hepatic artery supplying the right lobe of the liver only. The proper hepatic artery is missing and the left hepatic artery arises from the GDA. This variation does not correspond to any types of Michels' classification. (Folia Morphol 2014; 73, 4: 531-535)
\end{abstract}

Key words: hepatic arteries, variations, multidetector computed tomography angiography

\section{INTRODUCTION}

The multidetector-row computed tomography (CT) provides sufficient image quality for assessment of celiac trunk (CTr) variations and hepatic vessels anatomy and is commonly used in presurgical or postoperative evaluation of abdominal aorta $[4,11$, $13,20,21,25]$. The accurate preoperative evaluation of vascular pattern is of great clinical importance in hepatobiliary and pancreatic surgery, especially in hepatotransplantology and pancreatoduodenectomy
$[1,27]$. It is also essential for the successful therapy in patients undergoing endovascular surgical procedures such as embolisation of hepatic arteries or intra-arterial chemotherapy of hepatic neoplasm $[1,2,24]$. Aberrant hepatic arteries usually coexist with anatomical variations of the gastroduodenal artery (GDA). Variable origin and course of this artery results in complications during embolisation in case of bleeding duodenal cup ulcer [27]. It is also very important in planning hepatic arterial infusion che-

Address for correspondence: Dr A. Skórzewska, Department of Anatomy, University of Medical Sciences, ul. Święckickiego 6, 60-781 Poznań, Poland, tel: +48 6185465 48, e-mail: a.skorzewska@gmail.com 
motherapy when the GDA is used for the intraarterial chemotherapy pump placement $[2,23]$. The hepatic arterial infusion chemotherapy required an accomplishment of adequate distribution of anticancer agent in the liver parenchyma. It is very important to avoid the extrahepatic perfusion through inserted catheter [22, 23]. Inaccurate evaluation of arterial anatomy results failure of therapy due to incomplete drug distribution in the liver and vascular or gastrointestinal complication.

The anomalous arterial blood supply and exact description of anatomical variations is a challenge for radiologists. The purpose of this study was to find and analyse rare variations which are not described in Mithels' classification.

\section{MATERIALS AND METHODS}

Anatomical variations of hepatic arterial system were studied in 163 patients who underwent multidetector CT (MDCT) angiography in Department of General and Interventional Radiology Poznan University of Medical Sciences between April 2014 and June 2014. All examinations were clinically indicated routine diagnostic procedures with patients' permission and were performed on 64-row detector CT scanner (Light Speed, General Electric). Images were obtained from the level of diaphragm to the pubic symphysis. Slice thickness was $0.67 \mathrm{~mm}$; the pitch amounted to 1.3 with gantry rotation $0.5 \mathrm{~s}$. In all patients $100 \mathrm{~mL}$ of iodinate non-ionic contrast medium was injected into the brachial vein with an automatic syringe. Flow rate of contrast medium administration was $4.5 \mathrm{~mL} / \mathrm{s}$. It was followed by "wash out bolus" — injection of $50 \mathrm{~mL}$ of physiological salt solution. The examinations were evaluated in early arterial phase only (15-20 s after injection of contrast agent). Scans were analysed using General Electric workstation. Images were assessed using 2- and 3-dimensional reconstructions (maximum intensity projection - MIP; volume rendering - VR, auto bone extraction general). The pattern of hepatic arteries was classified according to Mitchels.

\section{RESULTS}

The typical hepatic anatomy was observed in 115 (70.6\%) patients, and 45 (27.6\%) patients demonstrate different types according to Mitchels. In 3 cases rare hepatic arteries variations coexisting with variable origin of the GDA were revealed.

The first variation was described in 65-year-old male who underwent MDCT angiography in the course of diagnosis of aneurysm by anastomosis after

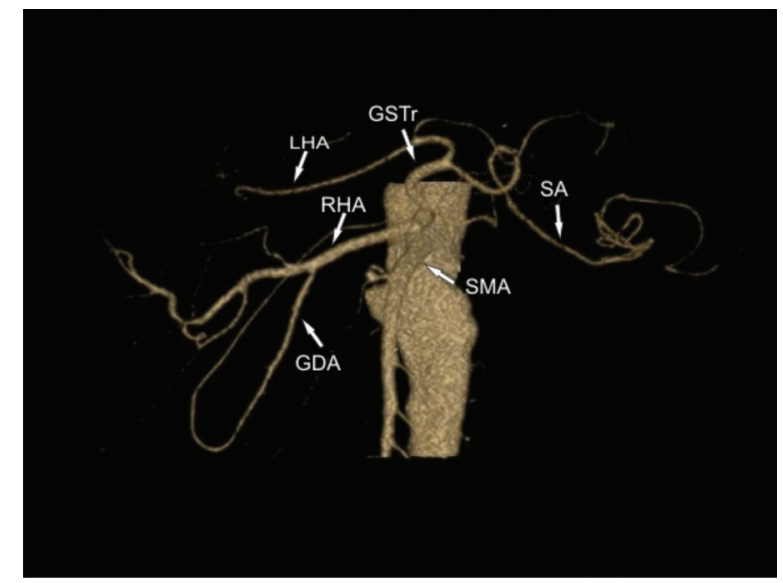

Figure 1. A 65-year-old man with aneurysm by anastomosis after surgical implantation of aorto-aortic tube prosthesis. The 3-dimensional volume rendered image shows the common hepatic artery originating from the celiac trunk and supplying right hepatic lobe. The left lobe is supplied by the left hepatic artery (LHA) arising from the gastrosplenic trunk (GSTr). The right hepatic artery (RHA) gives rise to the gastroduodenal artery (GDA). Superior mesenteric artery (SMA) originates from the abdominal aorta; SA — splenic artery.

surgical implantation of aorto-aortic tube prosthesis. The MDCT confirmed presence of aneurysm and revealed anatomical variation of $\mathrm{CTr}$ and hepatic arteries. The short arterial trunk arose from abdominal aorta as a first unpaired visceral branch from its anterior aspect, in the location typical for CTr. This trunk divided into 2 branches. One passing upward gave rise to the splenic artery (SA) and the left gastric artery (LGA), and finally supplied the left lobe of the liver. It was recognised as the left hepatic artery (LHA). The second branch of the common arterial trunk running to the right gave rise to the GDA. It supplied the right lobe of the liver and basing on the pattern of its ramification was considered to be the right hepatic artery (RHA) (Fig. 1).

The second variation was revealed in a 67-year-old male examined in MDCT angiography for suspicion of endoleak after endovascular aneurysm repair. Three years ago he underwent aortic stentgraft implantation. It was found in MDCT that the CTr divides into 4 branches: the SA, the LGA, and 2 arteries supplying the liver. These arteries corresponding to the RHA and the LHA arose independently from the CTr. The GDA originated from the LHA.

The third variation was found in a 78-year-old male examined in MDCT angiography during diagnosis of abdominal aortic aneurysm before surgical treatment (Fig. 2). Typical pattern of unpaired bran- 


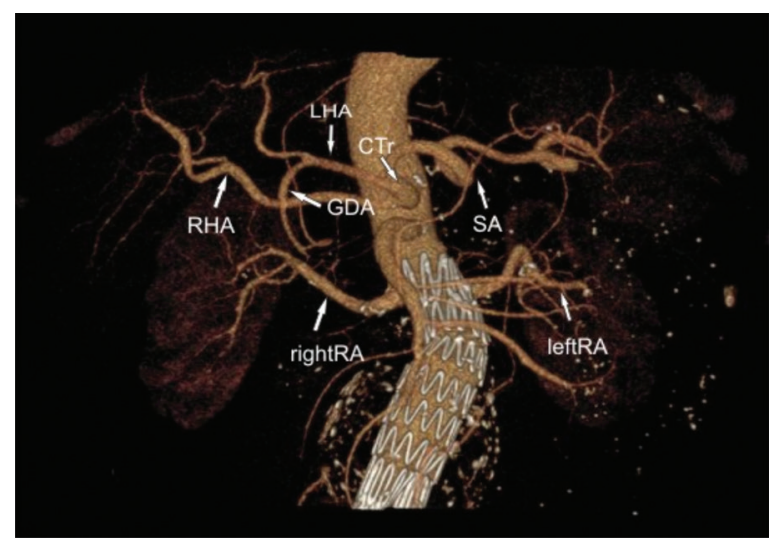

Figure 2. A 67-year-old man after endovascular aneurysm repair and aortic stentgraft implantation. The 3-dimensional volume rendered image demonstrates the right hepatic artery (RHA) and the left hepatic artery (LHA) originating independently from celiac trunk (CTr). The gastroduodenal artery (GDA) arises from the LHA; SA — splenic artery, left RA — left renal artery, right RA — right renal artery.

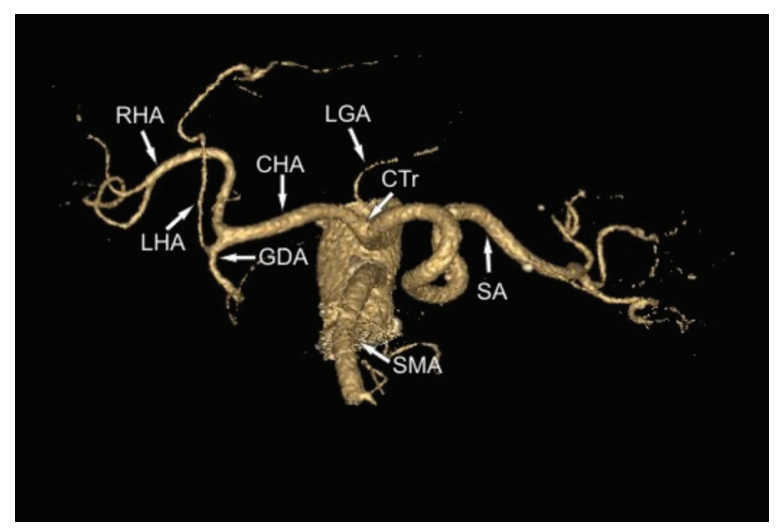

Figure 3. A 78-year-old man diagnosed for suspicion of abdominal aortic aneurysm. The 3 -dimensional volume rendered image reveals typical pattern of unpaired branches of abdominal aorta. The common hepatic artery (CHA) splits into the gastroduodenal artery (GDA) and the right hepatic artery (RHA). The GDA gives rise to the left hepatic artery (LHA); CTr — celiac trunk; LGA — left gastric artery; SA — splenic artery; SMA — superior mesenteric artery.

ches of the abdominal aorta was observed: the $\mathrm{CTr}$ divided into the SA, the LGA, and the common hepatic artery (CHA), and independent origin of the superior mesenteric artery (SMA). The CHA split into the GDA and the RHA typically branching in the right hepatic lobe. After a short course the GDA gave rise to the LHA which demonstrated typical pattern of its intrahepatic branches in the left lobe of the liver (Fig. 3).

\section{DISCUSSION}

Numerous variations of the hepatic arteries were described. According to the most commonly used
Mitchels' classification [15, 16] 10 types of them are distinguished depending on the origin of the lobar hepatic arteries and appearance of accessory hepatic arteries. The source of aberrant hepatic arteries is the LGA or the SMA.

According to Covey et al. [5] standard hepatic anatomy was observed in $61.3 \%$ of patients undergoing angiographic examination, and $19.8 \%$ of patients demonstrated variations of the origin and course of the LHA, and in $14.8 \%$ variations of the RHA were observed, while both aberrant hepatic arteries were found in $4.0 \%$ only.

The anatomical variations may be explained based on the embryonic development. According to Douard at al. [6] the liver anlage in human embryo is composed of 3 parts: right lateral, left lateral, and middle. They are connected by the median bridge and supplied by 3 primary embryonic hepatic arteries: right, middle, and left. In $51-74 \%$ of cases only one of them, primary middle hepatic artery (MHA), persists and transforms into the CHA typically branching into the GDA and the hepatic artery proper. The primary RHA usually disappears and the primary LHA transforms into the LGA. Finally the typical pattern of blood supply of the liver by 2 lobar branches of the hepatic artery proper is established. This pattern corresponds to Michels' the type 1 [16].

Two primary hepatic arteries persist in $20-25 \%$, and three of them in 2-12.5\% of cases [6]. They appear as the "accessory" and/or "replaced" hepatic arteries. The artery supplying the whole lobe of the liver and originating from the SMA or from the LGA is called replaced hepatic artery. The accessory hepatic artery supplies only the part of the lobe and exists as additional source of blood supply from the SMA or the LGA. Variations including replaced hepatic arteries were classified by Michels [16] as the type 2 (left replaced), type 3 (right replaced) and type 4 (both of them). Accessory hepatic arteries correspond to type 5 (left accessory), type 6 (right accessory) or type 7 (both). Type 8 is subdivided into $8 a, 8 b$, and $8 c$, and includes variations with coexisting replaced and accessory hepatic arteries. Vary rare condition is complete regression of the embryonic MHA and liver blood supply by RHA from the SMA or LHA from the LGA, or both. It corresponds to the type 9 or 10 .

Michels' classification was reduced to six types by Hiatt et al. [9] who classified as the same type both replaced and accessory hepatic arteries supplying the same lobe of the liver. It is emphasised that the 
exact definition of the CHA is essential to describe variations of hepatic arteries for practical purposes. Song at al. [24] proposed to name the CHA each artery that supplies at least one hepatic segment and gives raise to the GDA irrespective of its origin and course (not only this one that originates from the CTr). Chen et al. [3] presented their classification of hepatic arterial blood supply into 7 types based on the variable origin of the $\mathrm{CHA}$.

The persisted embryonic MHA usually supplies by its segmental branch the quadrate lobe of the liver $[6,10]$. The segmental branch for quadrate lobe is called in literature the MHA and, according to Wang et al. [26], exists in $71 \%$. It never originates from replaced hepatic arteries, regardless to their origin. As it was confirmed by Milon's studies [17], each hepatic artery that terminates as the MHA also gives rise to the GDA and is the CHA according to Song's et al. definition [24]. However another rare variation was observed by Adamthwaite et al. [1] during pancreatoduodenectomy. They found distal quadrifurcation of the CHA originating typically from the $\mathrm{CTr}$ and dividing into the RHA and LHA, and the intermediate branch, and right accessory hepatic artery. The intermediate branch supplied segment IV. The GDA arose from the accessory RHA.

In rare cases the liver is supplied from 3 sources. Gurgacz et al. [8] described 3 hepatic arteries: the MHA arising from $C T$, and 2 replaced arteries originating from the LGA and the SMA. In this case the MHA and the GDA originated from the CHA. Hlaing et al. [10] also observed 3 hepatic arteries: the right originating from the $\mathrm{CTr}$, and the left one from the LGA, and the CHA. The last one arose from the CTr and divided into the GDA and the MHA.

Three cases of total hepatic arterial blood supply derived from the LGA were found in MDCT by Okada et al. [19]. In these rare variants the GDA originated from the CHA or from the SA.

According to Song's et al. definition [24] the first described variation may be regarded as the $\mathrm{CHA}$ arising from the $\mathrm{CTr}$ and supplying the right hepatic lobe, whereas the left lobe is supplied by the LHA originating from gastrosplenic trunk. It may correspond to the type 2, according to Michels [15, 16], coexisting with one of 3 possible patterns of the $\mathrm{CTr}$ division (when the CHA is the first branch of the $\mathrm{CTr}$ and the gastrosplenic trunk is the second one).

The second case demonstrates the early bifurcation of the CHA just close to origin from the CTr. Both, the RHA and the LHA originate separately from the CTr. The GDA originates from the LHA that is more common variant as it was mentioned in literature [6]. The part of the LHA from its origin to the origin of the GDA may be named the CHA. This case corresponds to the variation of most common type 1 . As it is described in literature early bifurcation of the CHA, before the origin of the GDA, occurs in $6 \%$ of cases [6]. Usually the right branch originates as the first, and left branch is the source of the GDA being the $\mathrm{CHA}$ [8]. The last one also gives rise to the right gastric artery [8]. It is important to differentiate this variation with the accessory superior RHA originating from $\mathrm{CTr}$ and partially supplying the right lobe of the liver [7].

In the third case the CHA gives raise to the GDA and terminates as the RHA supplying the right lobe of the liver only. The proper hepatic artery is missing and the LHA arises from the GDA. This variation could not be classified according to Michels' classification.

Anatomical variations of the GDA were described by Milon et al. [17]. In rare cases the GDA originates directly from the $\mathrm{CT}$ and the $\mathrm{CHA}$ does not exist. Nayak et al. [18] describe 5 branches of the CTr: 3 typical, as well as the left phrenic artery, and the GDA. The gastroduodenosplenic trunk arising from the aorta was also mentioned in literature [14] and in this case the LGA and the accessory LHA arose directly from the abdominal aorta. According to Jones and Hardy [12] the RHA arises from the GDA in 6\% of cases. In $75 \%$ of cases it derives from the hepatic artery proper and only in $17 \%$ from the SMA.

\section{ACKNOWLEDGEMENTS}

The paper was supported by grant No. 502-14-020000000-071-47 from Poznan University of Medical Sciences.

\section{REFERENCES}

1. Adamthwaite JA, Pennington N, Menon KV (2007) Annomalous hepatic arierial anatomy discovered during pancreaticoduodenectomy. Surg Radiol Anat, 29: 269-271.

2. Allen PJ, Stojadinovic A, Ben-Porat L, Gonen M, Kooby D, Blumgart L, Paty P, Fong $Y$ (2002) The management of variant arterial anatomy during hepatic arterial infusion pump placement. Ann Surg Oncol, 9: 875-880.

3. Chen H, Yano R, Emura S, Shoumura S (2009) Anatomic variation of the celiac trunk with special reference to hepatic artery patterns. Ann Anat, 191: 399-407.

4. De Cecco CN, Ferrari R, Rengo M, Paolantonio P, Vecchietti F, Laghi A (2009) Anatomic variations of the hepatic arteries in 250 patients studied with 64-row CT angiography. Eur Radiol,19: 2765-2770. 
5. Covey AM, Brody LA, Maluccio MA, Getrajdman GI, Brown KT (2002) Variant hepatic arterial anatomy revisited: digital subtraction angiography performed in 600 patients. Radiol, 224: 542-547.

6. Douard R, Chevallier JM, Delmas V, Cugnenc PH (2006) Clinical interest of digestive arterial trunk anastomoses. Surg Radiol Anat, 28: 219-227.

7. Gielecki J, Żurada A, Sonpal N, Jabłońska B (2005) The clinical relevance of coeliac trunk variations. Folia Morphol, 3: 123-129.

8. Gurgacz AM, Horbaczewska A, Klimek-Piotrowska W, Walocha J (2011) Variations in hepatic vascularisation: lack of a proper hepatic artery. Two case reports. Folia Morphol, 70: 130-134.

9. Hiatt JR, Gabbay J, Bussutil RW (1994) Surgical anatomy of the hepatic arteries in 1000 cases. Ann Surg, 220: 50-52.

10. Hlaing KP, Othman F (2012) Complex pattern of a variant hepatic artery. Singapore Med J, 53: e186-e188.

11. lezzi R, RaVaele Cotroneo A, Marco Santoro DG, Storto ML (2008) Multidetector-row CT angiographic imaging of the celiac trunk: anatomy and normal variants. Surg Radiol Anat, 30: 303-310.

12. Jones RM, Hardy KJ, (2001) The hepatic artery: a reminder of surgical anatomy. JR Coll Surg Edinb, 46: 168-170.

13. Kornafel O, Baran B, Pawlikowska I, Laszczyński P, Guziński M, Sąsiadek M (2010) Analysis of anatomical variations of the main arteries branching from the abdominal aorta, with 64-detector computed tomography. Pol J Radiol, 75: 38-45.

14. Li J, Ren ZF (2011) Gastroduodenal-splenic trunk: an anatomical vascular variant. Rom J Morphol Embryol, 52: 1385-1387.

15. Michels NA (1955) Blood supply and anatomy of the upper abdominal organs with a descriptive atlas. JB Lippincot Co, Philadelphia, Montreal.

16. Michels NA (1966). Newer anatomy of the liver and its variant blood supply and collateral circulation. Am J Surg, 112: 337-347.

17. Milon J, Milon D, Boullier G, Le Guerrier A, Lanchou G (1978) The gastroduodenal artery and its variations. Study of 100 arteriographies. Bull Assoc Anatom, 62: 461-463.

18. Nayak SR, Prabhu LV, Krishnamurthy A, Ganesh Kumar C, Ramanathan LA, Acharya A, Prasad Sinha A (2008) Addi- tional branches of celiac trunk and its clinical significance. Romanian J Morphol Embryol, 49: 247-249.

19. Okada Y, Nishi N, Matsuo Y, Watadani T, Kimura F (2010) The common hepatic artery arising from the left gastric artery. Surg Radiol Anat, 32: 703-705.

20. Rountas C, Fanariotis M, Vlychou M, Arvanitis DL, Fezoulidis I, Vassiou K (2013) Celiomesenteric trunk demonstrated by multi-detector computed tomography angiography: two cases of a rare vascular variation. Folia Morphol, 2: 171-175.

21. Saba L, Mallarini G (2008) Multidetector row CT angiography in the evaluation of the hepatic artery and its anatomical variants. Clin Radiol, 63: 312-321.

22. Sahani DV, Krishnamurthy SK, Kalva S, Cusack J, Hahn PF, Santilli J, Saini S, Mueller PR (2004) Multidetector-row computed tomography angiography for planning intra-arterial chemotherapy pump placement in patients with colorectal metastases to the liver. J Comput Assist Tomogr, 4: 478-484.

23. Sone M, Kato K, Hirose A, Nakasato T, Tomabechi M, Ehara S, Hanari T (2008) Impact of multislice CT angiography on planning of radiological catheter placement for hepatic arterial infusion chemotherapy. Cardiovasc Intervent Radiol, 31: 91-97.

24. Song SY, Chung JW, Yin YH, Jae HJ, Kim HC, Jeon UB, Cho BH, So YH, Park JH (2010) Celiac axis and common hepatic artery variations in 5002 patients: systematic analysis with spiral CT and DSA. Radiol, 255: 278-288.

25. Ugurel MS, Battal B, Bozlar U, Nural MS, Tasar M, Ors F, Saglam M, Karademir I (2010) Anatomical variations of hepatic arterial system, coeliac trunk and renal arteries: an analysis with multidetector CT angiography. Br J Radiol, 83: 661-667.

26. Wang S, He X, Li Z, Peng Z, Tam NL, Sun C, Hu A, Huang J (2010) Characterization of the middle hepatic artery and its relevance to living donor liver transplantation. Liver Transpl, 16: 736-741.

27. Yang SH, Yin $Y H$, Jang J-Y, Lee SE, Chung JW, Suh K-S, Lee KU, Kim S-W (2007) Assessment of hepatic arterial anatomy in keeping with preservation of the vasculature while performing pancreatoduodenectomy: an opinion. World J Surg, 31: 2384-2391. 